\title{
Fast Predictive Post-OPC Contact/Via Printability Metric and Validation
}

\author{
Peng Yu and David Z. Pan \\ Electrical and Computer Engineering Department \\ The University of Texas at Austin
}

\begin{abstract}
Yield is one of the most important factors for massive semiconductor circuits production. As process variation tolerances decrease and the number of contacts/vias increase in modern technologies, contact/via failure has increased substantially, which attracts many attentions from both manufacture and design domains. Among all the contact/via failure mechanisms, lithography related ones become more important, the majority of which are rooted in focus and dose variations. Since the lithography image robustness is pattern dependent, conventional design rules are becoming less efficient and effective to convey the information. Models should be established to facilitate the evaluation of the lithography pattern robustness. Meanwhile, the models need to be fast enough to be used in design tools. Since Optical Proximity Correction (OPC) is very expensive to apply, the metric should be computed without doing actual OPC. We develop two new pre-OPC metrics to predict the post-OPC contact/via CD error due to focus variation, which are validated by our simulations. However, the metric for the post-OPC contact/via CD error due to dose variation is found not correlated well to the actual simulation. Further investigation is needed to increase the metric accuracy.
\end{abstract}

Keywords: Contact/via failure, lithography simulation, process variation, post-OPC prediction, printability

\section{INTRODUCTION}

Continuous semiconductor technology scaling has increased the functional complexity of integrated circuits (ICs), which results in the increase in the contact/via density and the number of metal layers. Due to the large number of contacts/vias in circuits, the failure in contact/via has already become an important yield limiting factor [1-6]. To reduce yield sensitivity to single via failure, the practice of redundant via insertion has been recommended in design rules [7].

There are many contact/via failure mechanisms, such as, due to current stress [8], thermal stress or electron migration $[9,10]$. Aside from these mechanisms, lithography related failures resulting from process variations have become more and more important for advanced technologies [11]. This is because lithography systems are susceptible to dose and focus variations, when the $k_{1}$ factor is small.

Due to the pattern dependent nature of these lithography related problems, it is possible to alleviate the problems in the design stage. The key is to develop a pattern goodness/fidelity metric. Conventionally, design rules have been used to convey this kind of manufacturing related information. However, rules become excessively complex as technology scales [12]. Model based metric can be the potential solution [13], but the metric should be computed without doing the actual OPC because OPC is very computation intensive [14]. There are a couple of pre-OPC metrics proposed for the litho-aware routing. In [15], the pre-OPC optical interference metric is used to guide OPC friendly routing. However, the interference is not a sound metric, because the interference can benefit OPC sometimes. In [16], the pre-OPC edge placement error (EPE) (i.e., the difference between the printed contour and the target) is used to screen out the lithography hotspots, which are then used to guide lithography-aware routing. Since OPC is always used in modern technologies, by the definition of EPE, the EPE after OPC (post-OPC EPE) should be THE metric for the image printability. The pre-OPC EPE at the nominal process condition used in [16] may not correlate well with the post-OPC EPE at the same process condition, since the post-OPC EPE is corrected by OPC and the EPE residue can be improved by better OPC algorithm and finer manufacturing grid, etc. What can not be improved by these methods is the post-OPC EPE variation (indicating

Further author information:

Peng Yu: E-mail: yupeng@cerc.utexas.edu

David Z. Pan: E-mail: dpan@ece.utexas.edu, Telephone: +1 (512) 471-1436 
the image robustness) due to process variations (such as dosage and focus). Our goal in this paper is to develop high-fidelity yet fast pre-OPC modeling for such post-OPC image robustness metrics under process variations.

A contact/via is to connect adjacent layers. The post-OPC contact/via size (also called Critical Dimension, or CD for short), which can be derived from EPE, indicates the goodness of the connection. We propose and validate three metrics to predict post OPC contact/via CD error due to process variations. The rest of this paper is organized as follows. Section 2 reviews the basics of lithography simulation and OPC algorithm that are used in the experiment. Section 3 discusses the new metrics. Section 4 shows the experimental validations. Section 5 concludes this paper.

\section{LITHOGRAPHY SIMULATION MODEL AND OPC RECIPE}

There are many variations between OPC software, such as different OPC recipes [14], and lithography simulation models used in these algorithms [17]. We use a common lithography simulation model and a typical OPC algorithm. More detailed descriptions can be found in [18] or its preliminary versions [19,20].

\subsection{Lithography Modeling Review}

\subsubsection{Diffused Latent Image}

Photoresist exposure and post-exposure-bake (PEB) steps cause nonuniform distribution of the chemicals in the photoresist. Although photoresist bulk is 3 -dimensional, it is commonly simplified as a 2-dimensional thin film in OPC softwares. $\mathcal{J}(\boldsymbol{k})$ is the chemical latent image in the Fourier domain can be written as

$$
\mathcal{J}(\boldsymbol{k})=\iint \mathcal{T}\left(\boldsymbol{k}+\boldsymbol{k}^{\prime}, \boldsymbol{k}^{\prime}\right) \mathcal{F}\left(\boldsymbol{k}+\boldsymbol{k}^{\prime}\right) \mathcal{F}^{*}\left(\boldsymbol{k}^{\prime}\right) \mathrm{d}^{2} \boldsymbol{k}^{\prime} .
$$

$\mathcal{F}(\boldsymbol{k})$ is the mask transmission function $F(\boldsymbol{r})$ in the Fourier domain, where $\boldsymbol{k}$ denotes a point in the frequency domain and $\boldsymbol{r}$ denotes a point in the spatial domain, which convention will be used in the following manuscript. $\mathcal{T}\left(\boldsymbol{k}, \boldsymbol{k}^{\prime}\right)$ is called the diffused transmission cross coefficient (DTCC), given by

$$
\mathcal{T}\left(\boldsymbol{k}^{\prime}, \boldsymbol{k}^{\prime \prime}\right)=\mathcal{G}\left(\boldsymbol{k}^{\prime}-\boldsymbol{k}^{\prime \prime}\right) \iint \mathcal{J}_{\mathrm{O}}^{-}(\boldsymbol{k}) \mathcal{K}\left(\boldsymbol{k}+\boldsymbol{k}^{\prime}\right) \mathcal{K}^{*}\left(\boldsymbol{k}+\boldsymbol{k}^{\prime \prime}\right) \mathrm{d}^{2} \boldsymbol{k},
$$

where the diffusion kernel $\mathcal{G}(\boldsymbol{k})$ corresponds the diffusion of the chemicals in the PEB step, $\mathcal{J}_{\mathrm{O}}^{-}(\boldsymbol{k})$ is the illumination function and $\mathcal{K}(\boldsymbol{k})$ is the projection system transfer function. The superscript * means the complex conjugation.

The diffusion kernel $\mathcal{G}(\boldsymbol{k})$ is written as

$$
\mathcal{G}(\boldsymbol{k})=e^{-2 \pi^{2} d^{2} k^{2}},
$$

where $d$ is the diffusion length and $k$ is the magnitude of $\boldsymbol{k}, k=|\boldsymbol{k}|$.

$\mathcal{K}(\boldsymbol{k})$ can be written as

$$
\mathcal{K}(\boldsymbol{k})=\mathcal{K}_{0}(\boldsymbol{k}) e^{i 2 \pi z \phi(\boldsymbol{k})},
$$

where $z$ denotes the focal error, and

$$
\phi(\boldsymbol{k})=\sqrt{1-k^{2}}
$$

is the phase factor. $\mathcal{K}_{0}(\boldsymbol{k})$ is the pupil filter. As an example, the pupil filter for a circle pupil (without lens aberrations) is

$$
\mathcal{K}_{0}(\boldsymbol{k})= \begin{cases}1 & k<1 \\ 0 & \text { otherwise }\end{cases}
$$

There are many illumination schemes. Table 1 lists a few commonly used ones. 
Table 1. Commonly used illumination schemes, where $A$ is a real normalization factor, which makes $\iiint_{\mathrm{O}}^{+\infty}(\boldsymbol{k}) \mathrm{d}^{2} \boldsymbol{k}=1$.

\begin{tabular}{|c|c|c|}
\hline Scheme & Parameters & Formulas \\
\hline Conventional & $0<\sigma<1$ & $\partial_{\mathrm{O}}^{-}(\boldsymbol{k})= \begin{cases}A & k<\sigma \\
0 & \text { otherwise. }\end{cases}$ \\
\hline Annular & $0<\sigma_{\text {in }}<\sigma_{\text {out }}<1$ & $\partial_{\mathrm{O}}^{-}(\boldsymbol{k})= \begin{cases}A & k>\sigma_{\text {in }} \text { and } k<\sigma_{\text {out }} \\
0 & \text { otherwise. }\end{cases}$ \\
\hline Normal quadrupole & \multirow{2}{*}{$\begin{array}{l}0<\sigma_{\text {radius }}<1 \text { and } \\
0<\sigma_{\text {center }}<1\end{array}$} & $\partial_{\mathrm{O}}^{-}(\boldsymbol{k})= \begin{cases}A & \left|\boldsymbol{k}-\sigma_{\text {center }}(\cos \theta, \sin \theta)\right|<\sigma_{\text {radius }} \text { and } k<1 \\
& \text { where } \theta=-135^{\circ},-45^{\circ}, 45^{\circ} \text { or } 135^{\circ}, \\
0 & \text { otherwise. }\end{cases}$ \\
\hline Cross quadrupole & & $\partial_{\mathrm{O}}^{-}(\boldsymbol{k})= \begin{cases}A & \left|\boldsymbol{k}-\sigma_{\text {center }}(\cos \theta, \sin \theta)\right|<\sigma_{\text {radius }} \text { and } k<1 \\
& \text { where } \theta=0^{\circ}, 90^{\circ}, 180^{\circ} \text { or } 270^{\circ}, \\
0 \quad \text { otherwise. }\end{cases}$ \\
\hline & Image Intensity & \\
\hline
\end{tabular}

Figure 1. Threshold bias model. The image intensity is denoted by the curve. A constant bias $B$ is applied the location where the intensity equals to the intensity threshold $I_{\text {th }}$ to get the printed contour.

\subsubsection{Threshold Bias Model}

Photoresist will be developed after photoresist exposure and PEB. To the first order of accuracy, we use the threshold bias photoresist model to predict the photoresist contour after development. Its simple form allows us to do further analysis. More sophisticated models shall be studied in the future. This model assumes that the printed contour can be computed by applying a constant bias $B$ to the contour where the intensity is equal to an intensity threshold $I_{\text {th }}$ (Figure 1 ). In the following discussion, we set $B$ to zero to simplify the discussion. The result can be extended easily to include $B \neq 0$ cases.

\subsubsection{Variational LIthography Model (VLIM)}

Exposure dose and focus variation are the two most important variations in lithography systems. Exposure dose variation can be modeled by varying the threshold $I_{\text {th }}$, which requires marginal additional computation once the image is computed. Since focus variation affect the image intensity profile, we review the variational lithography model, which simulates the image profile changes due to defocus efficiently.

The DTCC (2) can be expanded as the following

$$
\begin{aligned}
\mathcal{T}\left(\boldsymbol{k}^{\prime}, \boldsymbol{k}^{\prime \prime}\right)= & \mathcal{G}\left(\boldsymbol{k}^{\prime}-\boldsymbol{k}^{\prime \prime}\right) \sum_{n=0}^{\infty} \frac{(i 2 \pi z)^{n}}{n !} \sum_{m=0}^{n}\left(\begin{array}{c}
n \\
m
\end{array}\right) \iint \mathcal{J}_{O}^{-}(\boldsymbol{k}) \\
& \times\left(\phi\left(\boldsymbol{k}+\boldsymbol{k}^{\prime}\right)\right)^{m} \mathcal{K}_{0}\left(\boldsymbol{k}+\boldsymbol{k}^{\prime}\right)\left(-\phi\left(\boldsymbol{k}+\boldsymbol{k}^{\prime \prime}\right)\right)^{n-m} \mathcal{K}_{0}^{*}\left(\boldsymbol{k}+\boldsymbol{k}^{\prime \prime}\right) \mathrm{d}^{2} \boldsymbol{k} \\
= & \sum_{n=0}^{\infty} z^{n} \mathcal{T}_{n}\left(\boldsymbol{k}^{\prime}, \boldsymbol{k}^{\prime \prime}\right) .
\end{aligned}
$$


Plug (7) into (1), we end up with the following form

$$
\mathcal{J}(\boldsymbol{k})=\sum_{n=0}^{\infty} z^{n} \mathcal{J}_{n}(\boldsymbol{k}),
$$

where

$$
\mathcal{J}_{n}(\boldsymbol{k})=\iint \mathcal{T}_{n}\left(\boldsymbol{k}^{\prime}+\boldsymbol{k}, \boldsymbol{k}^{\prime}\right) \mathcal{F}\left(\boldsymbol{k}^{\prime}+\boldsymbol{k}\right) \mathcal{F}^{*}\left(\boldsymbol{k}^{\prime}\right) \mathrm{d}^{2} \boldsymbol{k}^{\prime}
$$

In the spatial domain, (8) becomes

$$
I(\boldsymbol{r})=\sum_{n=0}^{\infty} z^{n} I_{n}(\boldsymbol{r}) .
$$

For binary mask or PSM with phase $0^{\circ}$ and $180^{\circ}$ (the mask transmission function $F(\boldsymbol{r})$ is always real), we have the diffused latent image defocus expansion

$$
I(\boldsymbol{r})=\sum_{n=0}^{\infty} z^{2 n} I_{2 n}(\boldsymbol{r}),
$$

where the odd terms are all zero. When $z$ is small enough such that the higher order terms can be ignored, we have

$$
I(\boldsymbol{r}) \cong I_{0}(\boldsymbol{r})+z^{2} I_{2}(\boldsymbol{r})
$$

for the defocused image.

\subsection{OPC Algorithm and OPC Recipe for Vias}

Since sparse OPC algorithms [21] are still most commonly used in industry, we employ a sparse OPC algorithm for our experiments. In general, these algorithms consist of three parts:

- Segmentation: The edges of drawn polygon layout (target contour) are broken into smaller segments. These segments can be shifted perpendicular to its orientation (along the control line) during the segment movement step. This representation allows mask shapes be flexibly manipulated.

- Lithography simulation: The printed contour is simulated, which will be used to guide how the segments should be shifted.

- Segment movement: The segments are shifted accordingly to make the printed contour assimilate the target contour.

Figure 2 shows the meaning of the terminology used above for a contact/via. A contact/via is segmented into four segments. We take the center of each edge as the tagging point. We compute the intensity along the control line. Edge Placement Error (EPE), the difference between printed contour and the target contour, is computed along these lines.

We use constant ratio segment movement algorithm (Algorithm 1). Each segment is moved based on the EPE at its control point. We update the displacements of all the segments at the same time. It is an iterative algorithm, where the constant $C$ controls the edge movement step. 


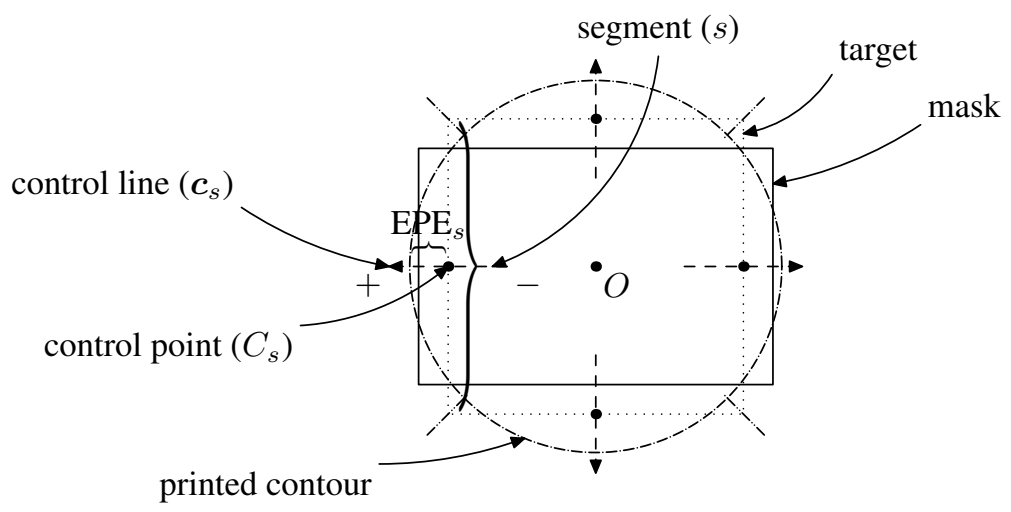

Figure 2. Contact/via segmentation and tagging. Each contact/via is segmented into four segments. The center point of each segment is the control point of the segment. The control lines are drawn perpendicular to the segment through the control points. Each segment can be shifted in the control line direction $(+$ or -$)$. EPE is computed along the control line. The segment displacement at one iteration depends on its current EPE. Shift of segment at the corner affects the length of the segment on the other side of the corner. $O$ is the center of the target.

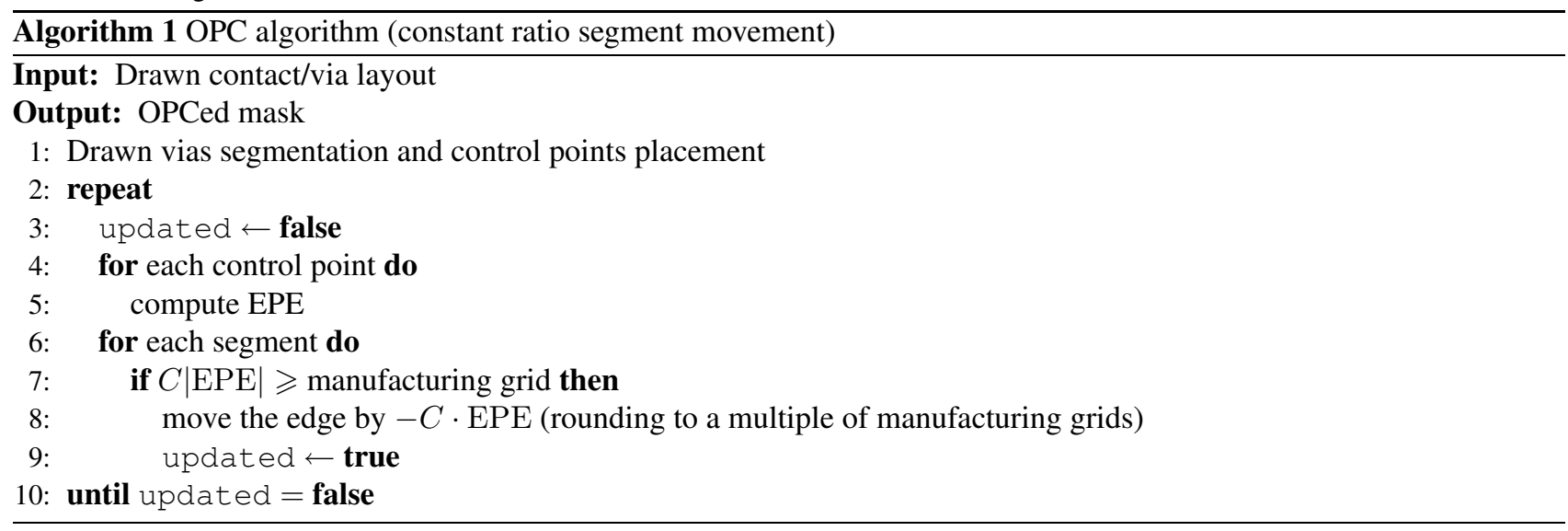

\section{POST OPC CONTACT/VIA CD PREDICTION}

We propose two pre-OPC metrics to estimate the post-OPC CD error due to focus variation. One is slower but more accurate than the other according to our experiment as we shall shown in Section 4. We also propose a metric to estimate the post-OPC CD error due to dose variation, which is not as good as those for focus variation.

As focus and exposure dose variations are the two most important process variations and the dose is related to $I_{\text {th }}$ (Section 2.1.3), we write CD as a function of the focus error $z$ and the intensity threshold $I_{\text {th }}, \mathrm{CD}_{\text {post }}\left(z, I_{\text {th }}\right)$. We denote the target $\mathrm{CD}$ as $\mathrm{CD}_{t}$. The post-OPC CD error at the process condition $\left(z, I_{\mathrm{th}}\right)$ can be decomposed into

$$
\mathrm{CD}_{\text {post }}\left(z, I_{\mathrm{th} 0}\right)-\mathrm{CD}_{\mathrm{t}}=\left(\mathrm{CD}_{\text {post }}\left(z, I_{\mathrm{th} 0}\right)-\mathrm{CD}_{\text {post }}\left(0, I_{\mathrm{th} 0}\right)\right)+\left(\mathrm{CD}_{\text {post }}\left(0, I_{\mathrm{th} 0}\right)-\mathrm{CD}_{\mathrm{t}}\right) .
$$

According to Line 7 of Algorithm 1, the second term on the right hand side of (13) is bounded by $\pm 2 \times$ grid size $/ C$, which depends on the OPC algorithm, but not on the process variations. Because the error bound of the second term can be improved by using better OPC algorithm or finer manufacturing grid, we only focus on the first term, which depends on the magnitude of the process variations.

\subsection{More Accurate But Slower Metric}

By the definition of EPE (see Figure 2), we have

$$
\mathrm{CD}_{\text {post }}\left(z, I_{\mathrm{th}}\right)=\mathrm{CD}_{t}+\frac{1}{2} \sum_{s=1}^{4} E_{s}\left(z, I_{\mathrm{th}}\right),
$$


where the summation is over the four segments of the contact/via and $E_{s}$ denotes for the segment $s$. The EPE depends on the intensity profile in a neighborhood of the control point $C_{s}$. As shown in Figure 3, we approximate the intensity profile as a straight line in a neighborhood of the control point $C_{s}$. Then we have

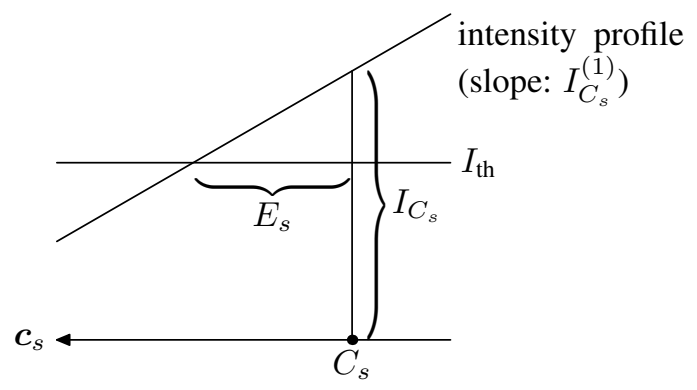

Figure 3. Intensity profile and EPE

$$
E_{s}=\frac{I_{C_{s}}-I_{\mathrm{th}}}{I_{C_{s}}^{(1)}}
$$

where $I_{C_{s}}$ and $I_{C_{s}}^{(1)}$ are the image intensity and the image intensity slope at the control point $C_{s}$. By taking the derivatives of (15), we have

$$
\frac{\partial E_{s}}{\partial I_{C_{s}}}=\frac{1}{I_{C_{s}}^{(1)}} \quad \text { and } \quad \frac{\partial E_{s}}{\partial I_{C_{s}}^{(1)}}=\frac{I_{\mathrm{th}}-I_{C_{s}}}{\left(I_{C_{s}}^{(1)}\right)^{2}} .
$$

The printed contour should be on target after OPC, which means $I_{C_{s}} \approx I_{\text {th }}$. Therefore we have $\frac{\partial E_{s}}{\partial I_{C_{s}}^{(1)}}=0$. By taking the derivative of $E_{s}$ with respect to $z^{2}$, we have *

$$
\frac{\partial E_{s}}{\partial\left(z^{2}\right)}=\frac{\partial E_{s}}{\partial I_{C_{s}}} \frac{\partial I_{C_{s}}}{\partial\left(z^{2}\right)}+\frac{\partial E_{s}}{\partial I_{C_{s}}^{(1)}} \frac{\partial I_{C_{s}}^{(1)}}{\partial\left(z^{2}\right)}=\frac{1}{I_{C_{s}}^{(1)}} \frac{\partial I_{C_{s}}}{\partial\left(z^{2}\right)} .
$$

With (17) and (14), we have

$$
\frac{\partial \mathrm{CD}_{\text {post }}}{\partial\left(z^{2}\right)}=\frac{1}{2} \sum_{s=1}^{4} \frac{1}{I_{C_{s}}^{(1)}} \frac{\partial I_{C_{s}}}{\partial\left(z^{2}\right)} .
$$

The right hand side of (18) is a post-OPC quantity, we use its pre-OPC counterpart in our metric. We define the pre-OPC metric $M_{1}$ as

$$
M_{1}(z)=\left.\frac{1}{2} \sum_{s=1}^{4} \frac{I_{C_{s}}}{I_{C_{s}}^{(1)}}\right|_{\mathrm{pre-OPC}, z} .
$$

The estimation of post-OPC CD difference $\left.\mathrm{CD}_{\text {post }}\right|_{z}-\left.\mathrm{CD}_{\text {post }}\right|_{0}$ is

$$
\left.\mathrm{CD}_{\text {post }}\right|_{z}-\left.\mathrm{CD}_{\text {post }}\right|_{0}=M_{1}(z)-M_{1}(0) \text {. }
$$

We could also take the derivative of $\mathrm{CD}_{\text {post-OPC }}$ with respect to $I_{\text {th }}$

$$
\frac{\partial \mathrm{CD}_{\mathrm{post}}}{\partial I_{\mathrm{th}}}=\frac{1}{2} \sum_{s=1}^{4} \frac{\partial E_{s}}{\partial I_{\mathrm{th}}},
$$

${ }^{*}$ Since the variational lithography modeling (12) shows that there is no $z^{1}$ term, we take the derivative with respect to $z^{2}$. 
which is the sensitivity of the CD with respected to $I_{\text {th }}$. We define the metric $M_{2}$ for the post-OPC CD sensitivity to $I_{\text {th }}$,

$$
M_{2}=\left.\frac{1}{2} \sum_{s=1}^{4} \frac{\partial E_{s}}{\partial I_{\mathrm{th}}}\right|_{\text {pre-OPC }}
$$

We will show in Section 4 that $M_{1}$ has good fidelity, but $M_{2}$ does not. Note that the post-OPC value of $M_{2}$ equals $\frac{\partial C D_{\text {post }}}{\partial I_{\text {th }}}$ by definition. The weak correlation between $M_{2}$ and $\frac{\partial \mathrm{CD}_{\text {post }}}{\partial I_{\text {th }}}$ means that $M_{2}$ is sensitive to OPC. We have to do actual OPC to estimate $\frac{\partial C D_{\text {post }}}{\partial I_{\text {th }}}$ better.

\subsection{Faster but Less Accurate Metric}

We are interested in small contacts/vias that are hard to print. In this case, the intensities around a contact/via are well correlated. Assuming the contact/via CD only depends on the intensity at the center $(O)$ of the contact/via $I_{O}$, we have

$$
\frac{\partial \mathrm{CD}_{\text {post }}}{\partial\left(z^{2}\right)}=\frac{\partial \mathrm{CD}}{\partial I_{O}} \frac{\partial I_{O}}{\partial\left(z^{2}\right)}
$$

Assuming the post-OPC intensity profiles are the same for all the contact/via pattern configurations ${ }^{\dagger}$, the intensity at the contact/via center $O$ at zero defocus (denoted as $\left.I_{O}\right|_{0}$ ) is the same for all the configurations as well, and the contact/via CD is linearly related to $I_{O}$ (meaning $\frac{\partial \mathrm{CD}}{\partial I_{O}}$ is a constant). We have

$$
\frac{\partial \mathrm{CD}_{\text {post }}}{\partial\left(z^{2}\right)} \propto \frac{1}{\left.I_{O}\right|_{0}} \frac{\partial I_{O}}{\partial\left(z^{2}\right)} .
$$

Then, we define the pre-OPC metric

$$
M_{3}(z)=\frac{\left.I_{O}\right|_{\mathrm{pre}, z}}{\left.I_{O}\right|_{\mathrm{pre}, 0}} .
$$

From (24) and (25), it is easy to see the estimation of post-OPC CD difference $\left.\mathrm{CD}_{\text {post }}\right|_{z}-\left.\mathrm{CD}_{\text {post }}\right|_{0}$ is

$$
\left.\mathrm{CD}_{\text {post }}\right|_{z}-\left.\mathrm{CD}_{\text {post }}\right|_{0} \propto M_{3}(z)-M_{3}(0)
$$

The metric $M_{3}$ requires two intensity computations, while $M_{1}$ requires at least eight intensity computations. Therefore, $M_{3}$ is a faster metric.

\section{EXPERIMENTAL RESULTS}

We use the numerical aperture $\mathrm{NA}=0.85$, the wavelength $\lambda=193 \mathrm{~nm}$ and the normal quadrupole illumination $\left(\sigma_{\text {center }}=\right.$ $0.8, \sigma_{\text {radius }}=0.3$ ), a typical illumination scheme for contact/via patterns. We randomly generate 1000 vias test patterns on a $5 \times 5$ lattice as shown in Figure 4. Each square denotes a contact/via site. The probability to put a contact/via at the solid square and the dashed square are $100 \%$ and $50 \%$ respectively. The lattice constant is $200 \mathrm{~nm}$. The target contact/via size is $100 \mathrm{~nm}$. We run OPC at the process condition $z=0 \mathrm{~nm}, I_{\mathrm{th}}=0.15$ with $C=0.5$. The manufacturing grid size is $1 \mathrm{~nm}$.

Figure 5 shows the average pre-OPC CD vs. post-OPC CD for the central contact/via in the lattice. The post-OPC CD error at nominal process condition is bounded by $\pm 2 \times$ grid size $/ C= \pm 4 \mathrm{~nm}$, as we mentioned in Section 3 .

Figure 6 and 7 show the experimental results for $M_{1}$ (see (20)) and $M_{3}$ (see (26)) respectively. The correlation coefficients for $M_{1}$ is bigger than that for $M_{3}$. So $M_{1}$ is a more accurate metric. Figure 8 shows that $M_{2}$ is not correlated well with $\frac{\partial C D_{\text {post }}}{\partial I_{\text {th }}}$. Therefore, $M_{2}$ can not be used to predict post OPC CD sensitivity to $I_{\text {th }}$.

\footnotetext{
${ }^{\dagger}$ The post-OPC CD equals to the target $\mathrm{CD}$. To the zeroth order approximation, the intensity profile are the same.
} 


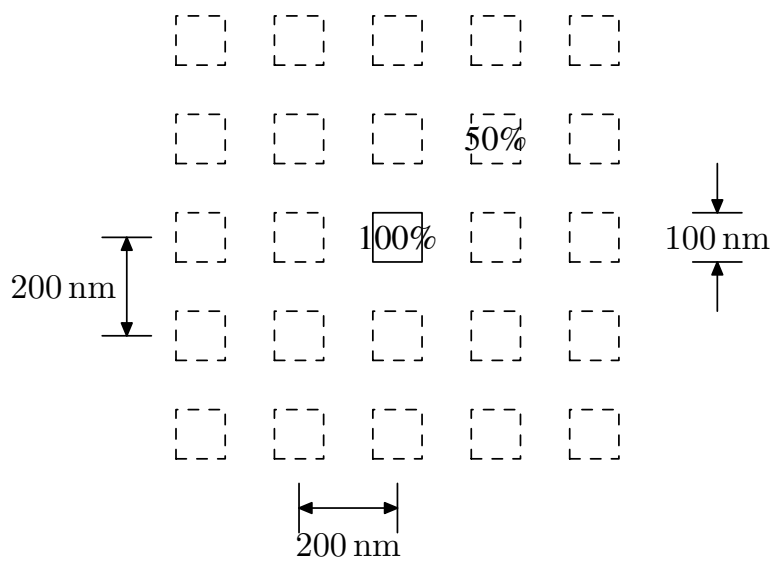

Figure 4. Contact/via lattice. The lattice constant is $200 \mathrm{~nm}$. A contact/via is always placed at the center solid square. A contact/via will be put at any dashed square with a probability of $50 \%$. The contact/via target size is $100 \mathrm{~nm}$.

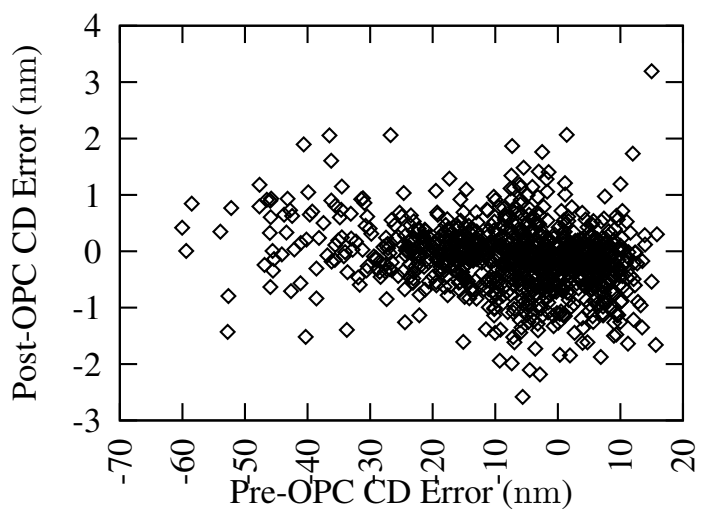

Figure 5. Pre- and post-OPC contact/via CD error relation for the center contact/via. CD is measured at the process condition $z=0$ and $I_{\text {th }}=I_{\text {th } 0}$ for both pre- and post- OPC. The post-OPC CD is bounded by $2 \times$ grid size $/ C=4 \mathrm{~nm}$.

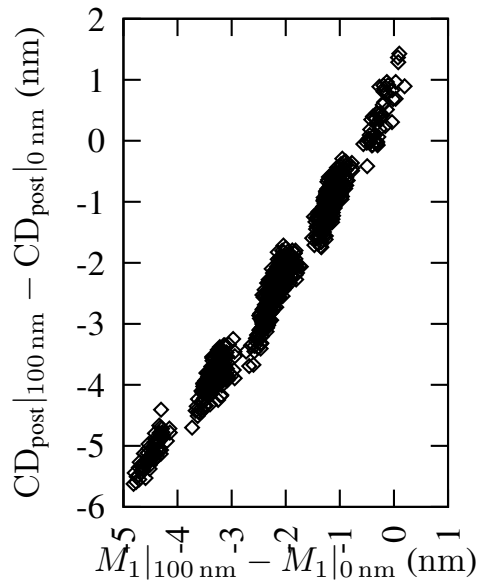

Figure 6. The more accurate but slower metric $M_{1}$. The correlation coefficient $R=0.9880$. 


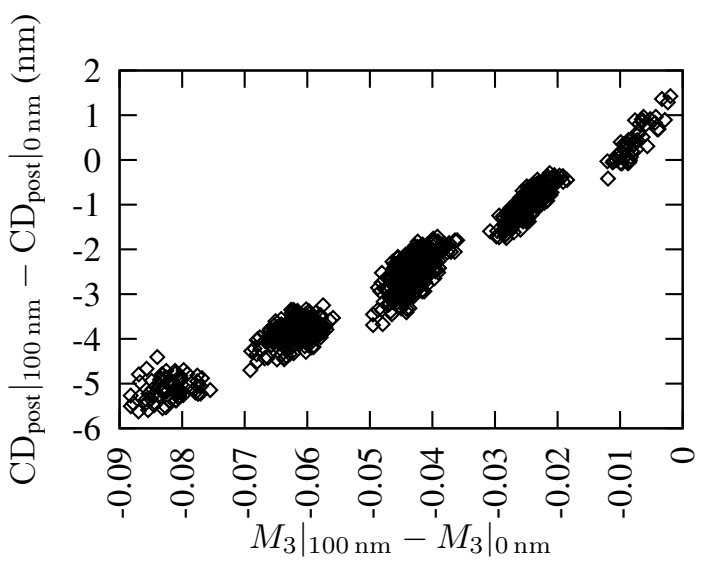

Figure 7. The faster but less accurate metric $M_{3}$. The correlation coefficient $R=0.9829$.

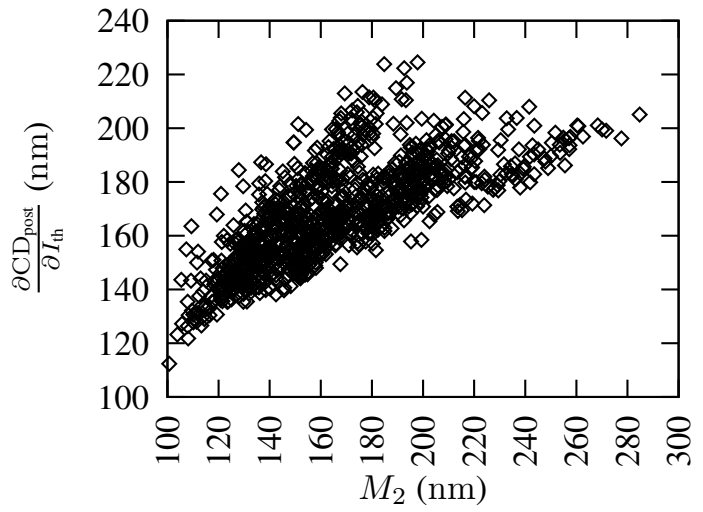

Figure 8. $M_{2}$ is not correlated well with CD sensitivity to $I_{\text {th }}$. The correlation coefficient $R=0.7406$.

\section{CONCLUSIONS}

After reviewing our lithography simulation model and OPC algorithm, we propose two pre-OPC metrics ( $M_{1}$ and $\left.M_{3}\right)$ to predict the post-OPC via $\mathrm{CD}$ variations due to focus variation. These metrics have different trade-offs between the accuracy and the speed. Our experiments validate these two metrics. The experiment result shows that the pre-OPC metrics $M_{2}$ to predict the post-OPC via CD variations due to dose variation is not as good as $M_{1}$ and $M_{3}$. Further investigation is needed to improve its accuracy.

\section{ACKNOWLEDGMENTS}

This work is partially sponsored by SRC, Fujitsu, IBM Faculty Award and Sun. We used computers donated by Intel Corporation and lithography simulation software $\left(\mathrm{PROLITH}^{\mathrm{TM}}\right)$ donated by KLA-Tencor.

\section{REFERENCES}

1. A. Kennen, J. Kornblum, J. Guravage, and L. Foster, "Investigation of high frequency failures on a $0.35 \mu \mathrm{m}$ CMOS IC," in Proc. ISTFA, pp. 359-364, Nov. 1999.

2. S. Venkataraman and S. B. Drummonds, "New techniques for logic fault diagnosis with a case study on the 440BX chipset," in Proc. ISTFA, pp. 389-396, Nov. 1999.

3. T. Kane, K. DeVries, M. Tenney, and A. Patel, "Characterization and fault identification of copper BEOL sub $0.25 \mu \mathrm{m}$ six level metal microprocessor designs," in Proc. ISTFA, pp. 335-341, Nov. 1999.

4. A. Gattiker, P. Nigh, and W. Maly, "Current-signature-based analysis of complex test fails," in Proc. ISTFA, pp. 377387, Nov. 1999. 
5. J.-Y. Glacet and F. Lee, "Embedded SRAM Bitmapping and Failure Analysis for Manufacturing Yield Improvement," in Proc. ISTFA, pp. 129-136, Nov. 2000.

6. S. W. Perungulam and G. K. S. Mekras, "Yield Enhancement Study: Process Variation and Design Margins Leading to Timing Issues in the RAM," in Proc. ISTFA, pp. 77-80, Nov. 2000.

7. Taiwan Semiconductor Manufacturing Company, Ltd, No. 9, Creation RD. 1, Science Based Industrial Park, HsinChu, Taiwan R.O.C, Design For Manufacturability Guide: Revised Redundant Via Insertion and ECO Route, Application Note, June 2004. TSMC Reference Flow Release 5.0.

8. K. Banerjee, A. Amerasekera, G. Dixit, N. Cheung, and C. Hu, "Characterization of contact and via failure under short durationhigh pulsed current stress," in Proc. IRPS, pp. 216-220, Nov. 1997.

9. A. Fischer, A. von Glasow, S. Penka, and F. Ungar, "Electromigration failure mechanism studies on copper interconnects," in Proc. IEEE IITC, pp. 139-141, 2002.

10. M. Lin, Y. Lin, J. Chen, C. Tsai, M.-S. Yeh, C. Liu, S. Hsu, C. Wang, Y. Sheng, K. Chang, K. Su, Y. Chang, and T. Wang, "The improvement of copper interconnect electromigration resistance by cap/dielectric interface treatment and geometrical design," in Proc. IEEE IRPS, pp. 229-233, Apr. 2004.

11. J. L. Sturtevant and D. Chou, "Toward full-chip prediction of yield-limiting contact patterning failure: correlation of simulated image parameters to advanced contact metrology metrics," in Proc. SPIE 6152, pp. 66-76, Apr. 2006.

12. M. Cote and P. Hurat, "Layout printability optimization using a silicon simulation methodology," in Proc. ISQED, pp. 159-164, 2004.

13. A. K. Wong, "Some Thoughts on the IC Design-Manufacture Interface," IEEE Des. Test 22(3), pp. 206-213, 2005.

14. N. Cobb and Y. Granik, "New concepts in OPC," in Proc. SPIE 5377, pp. 680-690, 2004.

15. L.-D. Huang and M. D. F. Wong, "Optical Proximity Correction (OPC)-Friendly Maze Routing," in Proc. DAC, pp. 186-191, 2004.

16. J. Mitra, P. Yu, and D. Z. Pan, "RADAR: RET-aware detailed routing using fast lithography simulations," in Proc. DAC, pp. 369-372, 2005.

17. Y. Granik, N. B. Cobb, and T. Do, "Universal process modeling with VTRE for OPC," in Proc. SPIE 4691, pp. 377394, July 2002.

18. P. Yu, S. X. Shi, and D. Z. Pan, "True Process Variation Aware Optical Proximity Correction with Variational Lithography Modeling and Model Calibration." submitted to Journal of Microlithography, Microfabrication, and Microsystems - Resolution Enhancement Techniques and Design for Manufacturability: Containing and Accounting for Variabilities in IC Creation, July 2007.

19. P. Yu, D. Z. Pan, and C. A. Mack, "Fast lithography simulation under focus variations for OPC and layout optimizations," in Proc. SPIE 6156, pp. 397-406, Apr. 2006.

20. P. Yu, S. X. Shi, and D. Z. Pan, "Process variation aware OPC with variational lithography modeling," in Proc. DAC, pp. 785-790, 2006.

21. N. B. Cobb, Fast Optical and Process Proximity Correction Algorithms for Integrated Circuit Manufacturing. PhD thesis, University of California at Berkeley, 1998. 